\title{
Robust Filtering for a Class of Stochastic Uncertain Nonlinear Time-Delay Systems via Exponential State Estimation
}

\author{
Zidong Wang, Member, IEEE, and Keith J. Burnham
}

\begin{abstract}
In this paper, we investigate the robust filter design problem for a class of nonlinear time-delay stochastic systems. The system under study involves stochastics, unknown state time-delay, parameter uncertainties, and unknown nonlinear disturbances, which are all often encountered in practice and the sources of instability. The aim of this problem is to design a linear, delayless, uncertainty-independent state estimator such that for all admissible uncertainties as well as nonlinear disturbances, the dynamics of the estimation error is stochastically exponentially stable in the mean square, independent of the time delay. Sufficient conditions are proposed to guarantee the existence of desired robust exponential filters, which are derived in terms of the solutions to algebraic Riccati inequalities. The developed theory is illustrated by numerical simulation.
\end{abstract}

Index Terms-Algebraic Riccati inequalities, nonlinear systems, robust filtering, stochastic exponential stability, time-delay systems.

\section{INTRODUCTION}

A S IS well known, for the purpose of analysis and control design, estimating the state variables of a dynamic model is important in heping to improve our knowledge about different systems. Hence, state estimation has been one of the fundamental issues in the control area. There have been a lot of works following those of Kalman (in the stochastic framework [1]) and Luenberger (in the deterministic one [23]), especially in signal processing applications.

One of the problems with Kalman filters, which has been well recognized now, is that the system under consideration has known dynamics described by a certain well-posed model, and its disturbances are Gaussian noises with known statistics. These assumptions limit the application scope of the Kalman filtering technique when there are uncertainties in either the exogenous input signals or the system model. It has been known that the standard Kalman filtering algorithms will generally not guarantee satisfactory performance when there exists uncertainty in the system model; see e.g., [1] and [6]. Motivated by

Manuscript received May 1, 2000; revised December 14, 2000. This work was supported in part by the University of Kaiserslautern of Germany and the Alexander von Humboldt Foundation of Germany. The associate editor coordinating the review of this paper and approving it for publication was Dr. Gonzalo Arce.

Z. Wang is with the Department of Mathematics, University of Kaiserslautern, Kaiserslautern, Germany and with the Control Theory and Applications Centre, School of Mathematical and Information Sciences, Coventry University, Coventry, U.K. (e-mail: zwang@mathematik.uni-kl.de).

K. J. Burnham is with the Control Theory and Applications Centre, School of Mathematical and Information Sciences, Coventry University, Coventry, U.K. (e-mail: K.Burnham@coventry.ac.uk).

Publisher Item Identifier S 1053-587X(01)02245-0. this problem, for the continuous-time case, a number of papers have attempted to extend the classical Kalman filter to systems involving norm-bounded uncertainties with respect to various filtering performance criteria, such as the $H_{\infty}$ specification, the minimum variance requirement, and the so-called admissible variance constraint. For the $H_{\infty}$ specification [3], [7], [9], [12], [15], [21], [28], [30], the $H_{\infty}$ norm of the transfer function from the noise input to the estimation error is minimized. By the minimum variance requirement [4], [5], [8], [10], [24], [25], [29], we mean that a minimal upper bound to the quadratic cost is guaranteed in spite of parameter uncertainties. Concerning the admissible variance constraint [31]-[33], [35], [36], the estimation error variance is required to be not more than the individual prespecified value, and the resulting design freedom is used to achieve other expected requirements $\left(H_{\infty}\right.$ performance, transient property, etc.).

On the other hand, it turns out that the delayed state is very often the cause for instability and poor performance of systems [18]. Increasing interests have recently been devoted to the robust and/or $H_{\infty}$ controller design problems of the linear uncertain state delayed systems. A great many papers have appeared on this topic; see [22] for a survey. However, the "dual" filter/observer design problems of uncertain time-delay systems have received much less attention, although they are important in control design and signal processing applications. In [34], the robust $H_{\infty}$ observer design problem has been studied for deterministic time-delay systems. In the stochastic framework, the robust Kalman filter design problem has been investigated in [13] and [17] for linear continuous- and discrete-time cases, respectively. A finite upper bound on the error covariance has been guaranteed in [13] and [17]. It should be pointed out that in [13] and [17], only the asymptotical stability has been considered on the filtering process, and therefore, a possibly long convergence time (although the steady-state covariance is bounded) may lead to poor performance. Often, in practice, exponential stability is highly desired for filtering processes so that fast convergence and acceptable accuracy in terms of reasonable error covariance can be ensured. In addition, it is well known that the nonlinearities are often introduced in the form of nonlinear disturbances. Unfortunately, the results in [13] and [17] no longer hold when the system under consideration involves nonlinearities that are frequently encountered in practice.

A filter is said to be exponential if the dynamics of the estimation error is stochastically exponentially stable. The design of exponential fast filters for linear and nonlinear stochastic systems is also an active research topic; see, e.g., 
[26] and [37]-[39]. Thus far, there have been very few papers dealing with the exponential filter design problem for general stochastic time-delay systems. Moreover, it seems more challenging to consider the case where parameter uncertainty and nonlinear disturbance also exist in the system model, due to the complexity of such a problem. This motivates us to investigate the multiobjective filter design problem for stochastic uncertain time-delay systems with stochastic exponential stability constraints.

In this paper, we address the robust filter design problem for a class of nonlinear time-delay stochastic systems. The class of stochastic time-delay systems under study is described by a state-space model with real time-varying norm-bounded parameter uncertainties and nonlinear disturbances meeting the boundedness condition. Here, attention is focused on the design of a linear, delayless, uncertainty-independent state estimator such that for all admissible uncertainties and nonlinear disturbances, the dynamics of the estimation error is stochastically exponentially stable in the mean square, independent of the time delay. Sufficient conditions are proposed to guarantee the existence of desired robust exponential filters, which are derived in terms of the solutions to algebraic Riccati inequalities. We demonstrate the usefulness and applicability of the developed theory by means of a numerical simulation example.

Notation: The notations in this paper are quite standard. $\mathbb{R}^{n}$ and $\mathbb{R}^{n \times m}$ denote, respectively, the $n$-dimensional Euclidean space and the set of all $n \times m$ real matrices. The superscript " $T$ " denotes the transpose and the notation $X \geq Y$ (respectively, $X>Y$ ), where $X$ and $Y$ are symmetric matrices, which means that $X-Y$ is positive semi-definite (respectively, positive definite). $I$ is the identity matrix with compatible dimension. We let $h>0$ and $C\left([-h, 0] ; \mathbb{R}^{n}\right)$ denote the family of continuous functions $\varphi$ from $[-h, 0]$ to $\mathbb{R}^{n}$ with the norm $\|\varphi\|=$ $\sup _{-h \leq \theta \leq 0}|\varphi(\theta)|$, where $|\cdot|$ is the Euclidean norm in $\mathbb{R}^{n}$. If $A$ is a matrix, denote by $\|A\|$ its operator norm, i.e., $\|A\|=$ $\sup \{|A x|:|x|=1\}=\sqrt{\lambda_{\max }\left(A^{T} A\right)}$, where $\lambda_{\max }(\cdot)$ [respectively, $\left.\lambda_{\min }(\cdot)\right]$ means the largest (respectively, smallest) eigenvalue of $A . l_{2}[0, \infty]$ is the space of the square integrable vector. Moreover, let $\left(\Omega, \mathcal{F},\left\{\mathcal{F}_{t}\right\}_{t>0}, P\right)$ be a complete probability space with a filtration $\left\{\mathcal{F}_{t}\right\}_{t \geq 0}$ satisfying the usual conditions (i.e., the filtration contains all $P$-null sets and is right continuous). Denote by $L_{\mathcal{F}_{0}}^{p}\left([-h, 0] ; \mathbb{R}^{n}\right)$ the family of all $\mathcal{F}_{0}$-measurable $C\left([-h, 0] ; \mathbb{R}^{n}\right)$-valued random variables $\xi=\{\xi(\theta)$ : $-h \leq \theta \leq 0\}$ such that $\sup _{-h \leq \theta \leq 0} \mathbb{E}|\xi(\theta)|^{p}<\infty$, where $\mathbb{E}\{\cdot\}$ stands for the mathematical expectation operator with respect to the given probability measure $P$. Sometimes, the arguments of a function will be omitted in the analysis when no confusion can arise.

\section{PROBlem Formulation AND Assumptions}

We consider a class of nonlinear uncertain continuous-time state delayed stochastic system represented by

$$
\begin{aligned}
\dot{x}(t)= & (A+\Delta A(t)) x(t)+\left(A_{d}+\Delta A_{d}(t)\right) \\
& \cdot x(t-h)+D f(x(t))+E_{1} w(t) \\
x(t)= & \varphi(t), \quad t \in[-h, 0]
\end{aligned}
$$

together with the measurement equation

$$
y(t)=(C+\Delta C(t)) x(t)+E_{2} w(t)
$$

where

$$
\begin{array}{ll}
x(t) \in \mathbb{R}^{n} & \text { state; } \\
y(t) \in \mathbb{R}^{p} & \text { measurement output; } \\
f(\cdot): \mathbb{R}^{n} \rightarrow \mathbb{R}^{n_{f}} & \text { unknown nonlinear disturbance input; } \\
h & \text { unknown state delay; } \\
\varphi(t) & \text { continuous vector valued initial function. }
\end{array}
$$

Here, $w(t)$ is a zero mean Gaussian white noise process with covariance $I>0$. The initial state $x(0)$ has the mean $\bar{x}(0)$ and covariance $P(0)$ and is uncorrelated with $w(t)$. $A, A_{d}, D, E_{1}, C, E_{2}$ are known constant matrices with appropriate dimensions. $\Delta A(t), \Delta A_{d}(t), \Delta C(t)$ are real-valued time-varying matrix functions representing norm-bounded parameter uncertainties and satisfy

$$
\left[\begin{array}{l}
\Delta A(t) \\
\Delta C(t)
\end{array}\right]=\left[\begin{array}{l}
M_{1} \\
M_{2}
\end{array}\right] F(t) N_{1}, \quad \Delta A_{d}=M_{1} F(t) N_{2}
$$

where $F(t) \in \mathbb{R}^{i \times j}$ is a real uncertain matrix with Lebesgue measurable elements and meets

$$
F^{T}(t) F(t) \leq I
$$

and $M_{1}, M_{2}, N_{1}, N_{2}$ are known real constant matrices of appropriate dimensions that specify how the uncertain parameters in $F(t)$ enter the nominal matrices $A, A_{d}, C$. The uncertainties $\Delta A(t), \Delta A_{d}(t), \Delta C(t)$ are said to be admissible if both (2.4) and (2.5) are satisfied.

Remark 2.1: For brevity, we have omitted the known control input terms in (2.1) and (2.3) since it is well known that this does not affect the generality of the discussion on the filter design.

Remark 2.2: The parameter uncertainty structure as in (2.4) and (2.5) has been widely used in the problems of robust control and robust filtering of uncertain systems (see, e.g., [14], [25], [35], and the references therein). Many practical systems possess parameter uncertainties that can be either exactly modeled or overbounded by (2.5). Observe that the unknown matrix $F(t)$ in (2.4) can even be allowed to be state-dependent, i.e., $F(t)=F(t, x(t))$, as long as (2.5) is satisfied.

Remark 2.3: Note that the system (2.1)-(2.3) can be used to represent many important physical systems subject to inherent state delays, parameter uncertainties, deterministic nonlinear disturbances (which may result from linearization process of an originally nonlinear plant or may be an actual external nonlinear input), and stochastic exogenous noises with known statistics.

Throughout this paper, we make the following assumptions.

Assumption 2.1: The system matrix $A$ is asymptotically stable.

Assumption 2.2: The matrix $M_{2}$ is of full row rank.

Assumption 2.3: There exists a known real constant matrix $H \in \mathbb{R}^{n \times n}$ such that the unknown nonlinear vector function $f(\cdot)$ satisfies the following boundedness condition

$$
|f(x(t))| \leq|H x(t)|
$$

for any $x(t) \in \mathbb{R}^{n}$. 
It is noted that Assumption 2.2 does not lose any generality. In this paper, the full-order linear filter under consideration is of the form

$$
\dot{\hat{x}}(t)=G \hat{x}(t)+K y(t)
$$

where the constant matrices $G$ and $K$ are filter parameters to be designed.

Letting the error state be

$$
e(t)=x(t)-\hat{x}(t)
$$

it then follows from (2.1)-(2.3) and (2.7) that

$$
\begin{aligned}
\dot{e}(t)= & G e(t)+[(A+\Delta A(t))-K(C+\Delta C(t))-G] x(t) \\
& +\left(A_{d}+\Delta A_{d}(t)\right) x(t-h)+D f(x(t)) \\
& +\left(E_{1}-K E_{2}\right) w(t) .
\end{aligned}
$$

Now, define

$$
\begin{aligned}
x_{f}(t) & :=\left[\begin{array}{l}
x(t) \\
e(t)
\end{array}\right], \quad A_{f}:=\left[\begin{array}{cc}
A & 0 \\
A-G-K C & G
\end{array}\right] \\
A_{d f} & :=\left[\begin{array}{ll}
A_{d} & 0 \\
A_{d} & 0
\end{array}\right] \\
D_{f} & :=\left[\begin{array}{l}
D \\
D
\end{array}\right], \quad E_{f}:=\left[\begin{array}{c}
E_{1} \\
E_{1}-K E_{2}
\end{array}\right] \\
M_{f} & :=\left[\begin{array}{ll}
M_{1} \\
M_{1}-K M_{2}
\end{array}\right] \\
N_{f} & :=\left[\begin{array}{ll}
N_{1} & 0
\end{array}\right], \quad \Delta A_{f}(t):=M_{f} F(t) N_{f} \\
M_{d f} & :=\left[\begin{array}{l}
M_{1} \\
M_{1}
\end{array}\right] \\
N_{d f} & :=\left[\begin{array}{ll}
N_{2} & 0
\end{array}\right], \quad \Delta A_{d f}(t):=M_{d f} F(t) N_{d f} \\
F_{f} & :=\left[\begin{array}{ll}
I & 0
\end{array}\right] .
\end{aligned}
$$

Noting

$$
x(t)=F_{f} x_{f}(t)
$$

and combining (2.1)-(2.4) and (2.9), we obtain the following augmented system:

$$
\begin{aligned}
\dot{x}_{f}(t)= & \left(A_{f}+\Delta A_{f}(t)\right) x_{f}(t)+\left(A_{d f}+\Delta A_{d f}(t)\right) x_{f}(t-h) \\
& +D_{f} f\left(F_{f} x_{f}(t)\right)+E_{f} w(t) .
\end{aligned}
$$

Next, observe the augmented system (2.15), and let $x_{f}(t ; \xi)$ denote the state trajectory from the initial data $x_{f}(\theta)=\xi(\theta)$ on $-h \leq \theta \leq 0$ in $L_{\mathcal{F}_{0}}^{2}\left([-h, 0] ; \mathbb{R}^{2 n}\right)$. Clearly, the system (2.15) admits a trivial solution $x_{f}(t ; 0) \equiv 0$ corresponding to the initial data $\xi=0$. We introduce the following stability concepts.

Definition 2.1: For the system (2.15) and every $\xi \in L_{\mathcal{F}_{0}}^{2}\left([-h, 0] ; \mathbb{R}^{2 n}\right)$, the trivial solution is asymptotically stable in the mean square if

$$
\lim _{t \rightarrow \infty} \mathbb{E}\left|x_{f}(t ; \xi)\right|^{2}=0
$$

and is exponentially stable in the mean square if there exist constants $\alpha>0$ and $\beta>0$ such that

$$
\mathbb{E}\left|x_{f}(t ; \xi)\right|^{2} \leq \alpha e^{-\beta t} \sup _{-h \leq \theta \leq 0} \mathbb{E}|\xi(\theta)|^{2} .
$$

Definition 2.2: We say that the filter (2.7) is exponential (respectively, asymptotic) if, for every $\xi \in L_{\mathcal{F}_{0}}^{2}\left([-h, 0] ; \mathbb{R}^{2 n}\right)$, the corresponding augmented system (2.15) is exponentially stable in mean square (respectively, asymptotically stable in the mean square).

The objective of this paper is to design an exponential filter for the uncertain nonlinear time-delay system (2.1)-(2.3). More specifically, we are interested in seeking the filter parameters $G$ and $K$ such that for all admissible parameter uncertainties $\Delta A, \Delta A_{d}, \Delta C$ and the nonlinear disturbance input $f(x(t))$, the augmented system (2.15) is exponentially stable in the mean square, independent of the unknown time-delay $h$.

\section{Main Results AND PROOFS}

\section{A. Filter Analysis}

This subsection is devoted to the filter analysis problem. Specifically, assuming that the filter structure is known, we will study the conditions under which the estimation error is stochastically exponentially stable in the mean square.

The following theorem shows that the exponential stability of a given filter for the uncertain nonlinear time-delay system (2.1)-(2.3) can be guaranteed if a positive definite solution to a modified algebraic Riccati-like matrix inequality (quadratic matrix inequality) is known to exist. This theorem plays a key role in the design of the expected filters.

Theorem 3.1: Let the filter parameters $G$ and $K$ be given. If there exist positive scalars $\varepsilon_{1}, \varepsilon_{2}, \varepsilon_{3}, \varepsilon_{4}>0$ and a positive definite matrix $P>0$ such that the following matrix inequality

$$
\begin{aligned}
& A_{f}^{T} P+P A_{f}+P\left[\left(\varepsilon_{1}+\varepsilon_{2}\right) I+\varepsilon_{3} M_{f} M_{f}^{T}+\varepsilon_{4}^{-1} D_{f} D_{f}^{T}\right] P \\
& \quad+\varepsilon_{1}^{-1} A_{d f}^{T} A_{d f}+\varepsilon_{3}^{-1} N_{f}^{T} N_{f}+\varepsilon_{4}\left(H F_{f}\right)^{T}\left(H F_{f}\right) \\
& \quad+\lambda_{\max }\left(M_{d f}^{T} M_{d f}\right) \varepsilon_{2}^{-1} N_{d f}^{T} N_{d f}<0
\end{aligned}
$$

holds, where $A_{f}, A_{d f}, M_{f}, N_{f}, D_{f}, M_{d f}, N_{d f}, F_{f}$ are defined in (2.10)-(2.13) and $H$ is defined in (2.6), then the augmented system (2.15) is exponentially stable in the mean square for all admissible parameter uncertainties $\Delta A, \Delta A_{d}, \Delta C$ and nonlinear disturbance input $f(x(t))$, independent of the unknown time-delay $h$.

Proof: For simplicity, we make the definitions

$$
\begin{aligned}
A_{1}(t) & :=A_{f}+\Delta A_{f}(t)=A_{f}+M_{f} F(t) N_{f} \\
A_{1 d}(t) & :=A_{d f}+\Delta A_{d f}(t)=A_{d f}+M_{d f} F(t) N_{d f}
\end{aligned}
$$

and then the augmented system (2.15) can be rewritten as

$$
\begin{aligned}
\dot{x}_{f}(t)= & A_{1}(t) x_{f}(t)+A_{1 d}(t) x_{f}(t-h)+D_{f} f\left(F_{f} x_{f}(t)\right) \\
& +E_{f} w(t) .
\end{aligned}
$$


Fix $\xi \in L_{\mathcal{F}_{0}}^{2}\left([-h, 0] ; \mathbb{R}^{2 n}\right)$ arbitrarily, and write $x_{f}(t ; \xi)=$ $x_{f}(t)$. For $\left(x_{f}(t), t\right) \in \mathbb{R}^{2 n} \times \mathbb{R}_{+}$, we define the Lyapunov function candidate

$$
Y\left(x_{f}(t), t\right)=x_{f}^{T}(t) P x_{f}(t)+\int_{t-h}^{t} x_{f}^{T}(s) Q x_{f}(s) d s
$$

where $P$ is the positive definite solution to the matrix inequality (3.1), and $Q>0$ is defined by

$$
Q:=\varepsilon_{1}^{-1} A_{d f}^{T} A_{d f}+\lambda_{\max }\left(M_{d f}^{T} M_{d f}\right) \varepsilon_{2}^{-1} N_{d f}^{T} N_{d f}
$$

By Itô's formula (see, e.g., [20]), the stochastic derivative of $Y$ along a given trajectory is obtained as

$$
\begin{aligned}
\frac{d}{d t} Y & \left(x_{f}(t), t\right) \\
= & x_{f}^{T}(t)\left(A_{1}^{T}(t) P+P A_{1}(t)+Q\right) x_{f}(t) \\
& +x_{f}^{T}(t-h) A_{1 d}^{T}(t) P x_{f}(t) \\
& +x_{f}^{T}(t) P A_{1 d}(t) x_{f}(t-h)-x_{f}^{T}(t-h) Q x_{f}(t-h) \\
& +f^{T}\left(F_{f} x_{f}(t)\right) D_{f}^{T} P x_{f}(t)+x_{f}^{T}(t) P D_{f} f\left(F_{f} x_{f}(t)\right) \\
& +2 x_{f}^{T}(t) P E_{f} w(t) \\
= & x_{f}^{T}(t)\left(A_{f}^{T} P+P A_{f}+Q\right) x_{f}(t)+x_{f}^{T}(t)\left[\left(\Delta A_{f}(t)\right)^{T} P\right. \\
& \left.+P\left(\Delta A_{f}(t)\right)\right] x_{f}(t)+x_{f}^{T}(t-h) A_{d f}^{T} P x_{f}(t) \\
& +x_{f}^{T}(t) P A_{d f} x_{f}(t-h) \\
& +x_{f}^{T}(t-h)\left(\Delta A_{d f}(t)\right)^{T} P x_{f}(t) \\
& +x_{f}^{T}(t) P\left(\Delta A_{d f}(t)\right) x_{f}(t-h) \\
& -x_{f}^{T}(t-h) Q x_{f}(t-h)+f^{T}\left(F_{f} x_{f}(t)\right) D_{f}^{T} P x_{f}(t) \\
& +x_{f}^{T}(t) P D f f\left(F_{f} x_{f}(t)\right)+2 x_{f}^{T}(t) P E_{f} w(t) .
\end{aligned}
$$

Let $\varepsilon_{1}, \varepsilon_{2}, \varepsilon_{3}, \varepsilon_{4}$ be positive scalars. Then, the matrix inequality

$$
\begin{aligned}
& {\left[\varepsilon_{1}^{1 / 2} x_{f}^{T}(t) P-\varepsilon_{1}^{-1 / 2} x_{f}^{T}(t-h) A_{d f}^{T}\right]} \\
& \quad \cdot\left[\varepsilon_{1}^{1 / 2} x_{f}^{T}(t) P-\varepsilon_{1}^{-1 / 2} x_{f}^{T}(t-h) A_{d f}^{T}\right]^{T} \geq 0
\end{aligned}
$$

yields

$$
\begin{aligned}
& x_{f}^{T}(t-h) A_{d f}^{T} P x_{f}(t)+x_{f}^{T}(t) P A_{d f} x_{f}(t-h) \\
& \quad \leq \varepsilon_{1} x_{f}^{T}(t) P^{2} x_{f}(t)+\varepsilon_{1}^{-1} x_{f}^{T}(t-h) A_{d f}^{T} A_{d f} x_{f}(t-h) .
\end{aligned}
$$

Moreover, noting that $\Delta A_{d f}(t)=M_{d f} F(t) N_{d f}$ and $F^{T}(t) F(t)$ $\leq I$, it follows from

$$
\begin{aligned}
& \left(\Delta A_{d f}(t)\right)^{T}\left(\Delta A_{d f}(t)\right) \\
& \quad \leq \lambda_{\max }\left(M_{d f}^{T} M_{d f}\right) N_{d f}^{T}\left(F^{T}(t) F(t)\right) N_{d f} \\
& \quad \leq \lambda_{\max }\left(M_{d f}^{T} M_{d f}\right) N_{d f}^{T} N_{d f}
\end{aligned}
$$

and

$$
\begin{gathered}
\Psi_{1}:=\varepsilon_{2}^{1 / 2} x_{f}^{T}(t) P-\varepsilon_{2}^{-1 / 2} x_{f}^{T}(t-h)\left(\Delta A_{d f}(t)\right)^{T} \\
\Psi_{1} \Psi_{1}^{T} \geq 0
\end{gathered}
$$

that

$$
\begin{aligned}
& x_{f}^{T}(t-h)\left(\Delta A_{d f}(t)\right)^{T} P x_{f}(t)+x_{f}^{T}(t) P\left(\Delta A_{d f}(t)\right) x_{f}(t-h) \\
& \leq \varepsilon_{2} x_{f}^{T}(t) P^{2} x_{f}(t)+\varepsilon_{2}^{-1} \lambda_{\max }\left(M_{d f}^{T} M_{d f}\right) x_{f}^{T}(t-h) \\
& \quad \cdot N_{d f}^{T} N_{d f} x_{f}(t-h) .
\end{aligned}
$$

Next, it results from

$$
\begin{gathered}
\Psi_{2}:=\varepsilon_{3}^{1 / 2} P M_{f}-\varepsilon_{3}^{-1 / 2} N_{f}^{T} F^{T}(t), \quad \Psi_{2} \Psi_{2}^{T} \geq 0 \\
F^{T}(t) F(t) \leq I
\end{gathered}
$$

that

$$
\begin{aligned}
& x_{f}^{T}(t)\left[\left(\Delta A_{f}(t)\right)^{T} P+P\left(\Delta A_{f}(t)\right)\right] x_{f}(t) \\
& \quad=x_{f}^{T}(t)\left[\left(M_{f} F(t) N_{f}\right)^{T} P+P\left(M_{f} F(t) N_{f}\right)\right] x_{f}(t) \\
& \quad \leq x_{f}^{T}(t)\left[\varepsilon_{3} P M_{f} M_{f}^{T} P+\varepsilon_{3}^{-1} N_{f}^{T} F^{T}(t) F(t) N_{f}\right] x_{f}(t) \\
& \quad \leq \varepsilon_{3} x_{f}^{T}(t) P M_{f} M_{f}^{T} P x_{f}(t)+\varepsilon_{3}^{-1} x_{f}^{T}(t) N_{f}^{T} N_{f} x_{f}(t) .
\end{aligned}
$$

Furthermore, from

$$
\begin{aligned}
& {\left[\varepsilon_{4}^{1 / 2} f^{T}\left(F_{f} x_{f}(t)\right)-\varepsilon_{4}^{-1 / 2} x_{f}^{T}(t) P D_{f}\right]} \\
& \quad \cdot\left[\varepsilon_{4}^{1 / 2} f^{T}\left(F_{f} x_{f}(t)\right)-\varepsilon_{4}^{-1 / 2} x_{f}^{T}(t) P D_{f}\right]^{T} \geq 0
\end{aligned}
$$

and Assumption 2.3, we have

$$
\begin{aligned}
f^{T} & \left(F_{f} x_{f}(t)\right) D_{f}^{T} P x_{f}(t)+x_{f}^{T}(t) P D_{f} f\left(F_{f} x_{f}(t)\right) \\
\leq & \varepsilon_{4} f^{T}\left(F_{f} x_{f}(t)\right) f\left(F_{f} x_{f}(t)\right)+\varepsilon_{4}^{-1} x_{f}^{T}(t) P D_{f} D_{f}^{T} P x_{f}(t) \\
= & \varepsilon_{4} f^{T}(x(t)) f(x(t))+\varepsilon_{4}^{-1} x_{f}^{T}(t) P D_{f} D_{f}^{T} P x_{f}(t) \\
\leq & \varepsilon_{4}|H x(t)|^{2}+\varepsilon_{4}^{-1} x_{f}^{T}(t) P D_{f} D_{f}^{T} P x_{f}(t) \\
= & \varepsilon_{4} x^{T}(t) H^{T} H x(t)+\varepsilon_{4}^{-1} x_{f}^{T}(t) P D_{f} D_{f}^{T} P x_{f}(t) \\
= & \varepsilon_{4} x_{f}^{T}(t)\left(H F_{f}\right)^{T}\left(H F_{f}\right) x_{f}(t) \\
& +\varepsilon_{4}^{-1} x_{f}^{T}(t) P D_{f} D_{f}^{T} P x_{f}(t) .
\end{aligned}
$$

Noticing the inequality (3.1) and the definition (3.6), we denote

$$
\begin{aligned}
\Pi:= & A_{f}^{T} P+P A_{f} \\
& +P\left[\left(\varepsilon_{1}+\varepsilon_{2}\right) I+\varepsilon_{3} M_{f} M_{f}^{T}+\varepsilon_{4}^{-1} D_{f} D_{f}^{T}\right] P \\
& +\varepsilon_{1}^{-1} A_{d f}^{T} A_{d f}+\varepsilon_{3}^{-1} N_{f}^{T} N_{f}+\varepsilon_{4}\left(H F_{f}\right)^{T}\left(H F_{f}\right) \\
& +\lambda_{\max }\left(M_{d f}^{T} M_{d f}\right) \varepsilon_{2}^{-1} N_{d f}^{T} N_{d f}<0 .
\end{aligned}
$$

Then, substituting (3.8)-(3.11) into (3.7) results in

$$
\begin{aligned}
\frac{d}{d t} & Y\left(x_{f}(t), t\right) \\
& \leq x_{f}^{T}(t) \Pi x_{f}(t)+2 x_{f}^{T}(t) P E_{f} w(t) \\
& \leq-\lambda_{\min }(-\Pi) x_{f}^{T}(t) x_{f}(t) d t+2 x_{f}^{T}(t) P E_{f} w(t)
\end{aligned}
$$

which means that the nonlinear uncertain stochastic time-delay augmented system (2.15) is asymptotically stable (in the mean square), provided that the inequality (3.1) is met. 
Next, to show the expected exponential stability (in the mean square) of the augmented system, some standard manipulations will be made on (3.13) by exploiting the technique developed in [19] and [20].

Let $\beta$ be the unique root of the equation

$$
\lambda_{\min }(-\Pi)-\beta \lambda_{\max }(P)-\beta h \lambda_{\max }(Q) e^{\beta h}=0
$$

where $\Pi$ and $Q$ are defined, respectively, in (3.12) and (3.6), $P$ is the positive definite solution to (3.1), and $h$ is the unknown time delay.

We can obtain from (3.13) that

$$
\begin{aligned}
& d\left[e^{\beta t} Y\left(x_{f}(t), t\right)\right] \\
& =e^{\beta t}\left[\beta Y\left(x_{f}(t), t\right) d t+d Y\left(x_{f}(t), t\right)\right] \\
& \leq e^{\beta t}\left(-\quad\left[\lambda_{\min }(-\Pi)-\beta \lambda_{\max }(P)\right]\left|x_{f}(t)\right|^{2}\right. \\
& \left.\quad \quad+\beta h \lambda_{\max }(Q) \int_{t-h}^{t}\left|x_{f}(s)\right|^{2} d s\right) d t \\
& \quad+2 e^{\beta t} x_{f}^{T}(t) P E_{f} w(t) d t .
\end{aligned}
$$

Then, integrating both sides from 0 to $T>0$ and taking the expectation result in

$$
\begin{aligned}
& e^{\beta t} \mathbb{E} Y\left(x_{f}(T), T\right) \\
& \leq {\left[\lambda_{\max }(P)+h \lambda_{\max }(Q)\right] \sup _{-h \leq \theta \leq 0} \mathbb{E}|\xi(\theta)|^{2} } \\
&-\left[\lambda_{\min }(-\Pi)-\beta \lambda_{\max }(P)\right] \mathbb{E} \int_{0}^{T} e^{\beta t}\left|x_{f}(t)\right|^{2} d t \\
&+\beta \lambda_{\max }(Q) \mathbb{E} \int_{0}^{T} e^{\beta t} \int_{t-h}^{t}\left|x_{f}(s)\right|^{2} d s d t .
\end{aligned}
$$

Note that

$$
\begin{aligned}
& \int_{0}^{T} e^{\beta t} \int_{t-h}^{t}\left|x_{f}(s)\right|^{2} d s d t \\
& \quad \leq \int_{-h}^{T}\left(\int_{\max (s, 0)}^{\min (s+h, T)} e^{\beta t} d t\right)\left|x_{f}(s)\right|^{2} d s \\
& \quad \leq \int_{-h}^{T} h e^{\beta(s+h)}\left|x_{f}(s)\right|^{2} d s \\
& \quad \leq h e^{\beta h} \int_{0}^{T} e^{\beta t}\left|x_{f}(t)\right|^{2} d t+h e^{\beta h} \int_{-h}^{0}|\xi(\theta)|^{2} d \theta .
\end{aligned}
$$

Then, considering the definition of $\beta$ in (3.14), we have

$$
\begin{aligned}
& e^{\beta T} \mathbb{E} Y\left(x_{f}(T), T\right) \\
& \leq {\left[\lambda_{\max }(P)+h \lambda_{\max }(Q)\right] \sup _{-h \leq \theta \leq 0} \mathbb{E}|\xi(\theta)|^{2} } \\
&+\beta \lambda_{\max }(Q) h^{2} e^{\beta h} \sup _{-h \leq \theta \leq 0} \mathbb{E}|\xi(\theta)|^{2}
\end{aligned}
$$

and

$$
\begin{aligned}
\mathbb{E}\left|x_{f}(T)\right|^{2} & \\
\leq \lambda_{\min }^{-1}(P)( & {\left[\lambda_{\max }(P)+h \lambda_{\max }(Q)\right] \sup _{-h \leq \theta \leq 0} \mathbb{E}|\xi(\theta)|^{2} } \\
& \left.+\beta \lambda_{\max }(Q) h^{2} e^{\beta h} \sup _{-h \leq \theta \leq 0} \mathbb{E}|\xi(\theta)|^{2}\right) e^{-\beta h}
\end{aligned}
$$

Notice that $T>0$ is arbitrary, and letting

$$
\alpha:=\lambda_{\min }^{-1}(P)\left[\lambda_{\max }(P)+h \lambda_{\max }(Q)\left(1+h e^{\beta h}\right)\right]
$$

the definition of exponential stability in (2.17) is then satisfied, and this completes the proof of Theorem 3.1.

Remark 3.1: Theorem 3.1 offers the analysis results for the exponential stability (in the mean square) of a class of nonlinear uncertain time-delay stochastic systems. The results may be conservative due to the use of the inequalities (3.8)-(3.11). However, such conservativeness can be significantly reduced by appropriate choices of the parameters $\varepsilon_{1}, \varepsilon_{2}, \varepsilon_{3}, \varepsilon_{4}$ in a matrix norm sense. The relevant discussion and corresponding numerical algorithm can be found in [36] and references therein.

Remark 3.2: The result of Theorem 3.1 can be readily extended to the multiple state delayed case. Consider the following nonlinear uncertain continuous-time multidelay stochastic system:

$$
\begin{aligned}
\dot{x}(t)= & (A+\Delta A(t)) x(t)+\sum_{i=1}^{r}\left(A_{d i}+\Delta A_{d i}(t)\right) x\left(t-h_{i}\right) \\
& +D f(x(t))+E_{1} w(t) \\
x(t)= & \varphi(t), \quad t \in[-h, 0], \quad 0<h=\max _{i}\left(h_{i}\right)
\end{aligned}
$$

where the uncertainties satisfy

$$
\begin{aligned}
{\left[\begin{array}{l}
\Delta A(t) \\
\Delta C(t)
\end{array}\right] } & =\left[\begin{array}{l}
M_{1} \\
M_{2}
\end{array}\right] F(t) N_{1}, \\
\Delta A_{d i}(t) & =M_{1} F(t) N_{d i} \quad F^{T}(t) F(t) \leq I
\end{aligned}
$$

for $i=1,2, \cdots, r$. We may obtain an augmented system that is similar to (2.15). Then, instead of (3.5), we define the Lyapunov function

$$
Y\left(x_{f}(t), t\right)=x_{f}^{T}(t) P x_{f}(t)+\sum_{i=1}^{r} \int_{t-h_{i}}^{t} x_{f}^{T}(s) Q_{i} x_{f}(s) d s .
$$

Following the same line of the proof of Theorem 3.1, a parallel result can be easily obtained for the multidelay case. The reason why we discuss the single delay case is to make our theory more understandable and to avoid unnecessarily complicated notations.

The following corollary, which results easily from [20], reveals that for the linear delay stochastic system (2.15), the exponential stability in the mean square implies the almost surely exponential stability.

Corollary: Under the conditions of Theorem 3.1, the uncertain time-delay system (2.15) is almost surely exponentially stable in mean square for all admissible parameter uncertainties $\Delta A, \Delta A_{d}, \Delta C$ and nonlinear disturbance input $f(x(t))$, independent of the unknown time-delay $h$, i.e.,

$$
\lim _{t \rightarrow \infty} \sup \frac{1}{t} \log \left|x_{f}(t ; \xi)\right| \leq-\frac{\beta}{2}
$$

almost surely holds for all $\xi \in L_{\mathcal{F}_{0}}^{2}\left([-h, 0] ; \mathbb{R}^{2 n}\right)$, where $\beta>0$ is the unique root of (3.14).

\section{B. Filter Design}

This subsection is devoted to the design of filter parameters $G$ and $K$ by using the result in Theorem 3.1. We derive the explicit 
expressions of the expected filter parameters in terms of the positive definite solutions of two Riccati-like matrix inequalities.

The following lemma is easily accessible and will be used in the proofs of our main results in this paper.

Lemma 3.1: For a given negative definite matrix $\Upsilon<0(\Upsilon \in$ $\left.\mathbb{R}^{n \times n}\right)$, there always exists a matrix $S \in \mathbb{R}^{n \times p}(p \leq n)$ such that $\Upsilon+S S^{T}<0$.

Prior to stating the main results of this paper, we give the following definitions for the sake of simplicity:

$$
\begin{aligned}
\hat{A} & :=A+\varepsilon_{3} M_{1} M_{1}^{T} P_{1}+\varepsilon_{4}^{-1} D D^{T} P_{1} \\
\hat{C} & :=C+\varepsilon_{3} M_{2} M_{1}^{T} P_{1} \\
R & :=\varepsilon_{3} M_{2} M_{2}^{T} \\
\Theta & :=\hat{C}+\varepsilon_{3} M_{2} M_{1}^{T} P_{2} .
\end{aligned}
$$

The following theorem shows that the desired filter parameters can be obtained in terms of the positive definite solutions to two quadratic matrix inequalities (QMIs).

Theorem 3.2: If there exist positive scalars $\varepsilon_{1}, \varepsilon_{2}, \varepsilon_{3}, \varepsilon_{4}>$ 0 such that the following two QMIs

$$
\begin{aligned}
A^{T} P_{1}+P_{1} A+ & P_{1}\left[\left(\varepsilon_{1}+\varepsilon_{2}\right) I+\varepsilon_{3} M_{1} M_{1}^{T}+\varepsilon_{4}^{-1} D D^{T}\right] P_{1} \\
& +2 \varepsilon_{1}^{-1} A_{d}^{T} A_{d}+\varepsilon_{3}^{-1} N_{1}^{T} N_{1}+\varepsilon_{4} H^{T} H \\
& +\lambda_{\max }\left(M_{d f}^{T} M_{d f}\right) \varepsilon_{2}^{-1} N_{2}^{T} N_{2}<0 \\
\Upsilon:= & \left(\hat{A}-\varepsilon_{3} M_{1} M_{2}^{T} R^{-1} \hat{C}\right)^{T} P_{2} \\
& +P_{2}\left(\hat{A}-\varepsilon_{3} M_{1} M_{2}^{T} R^{-1} \hat{C}\right) \\
+ & P_{2}\left[\left(\varepsilon_{1}+\varepsilon_{2}\right) I+\varepsilon_{3} M_{1} M_{1}^{T}+\varepsilon_{4}^{-1} D D^{T}\right. \\
& \left.-\varepsilon_{3}^{2} M_{1} M_{2}^{T} R^{-1} M_{2} M_{1}^{T}\right] \\
& \cdot P_{2}-\hat{C}^{T} R^{-1} \hat{C}<0
\end{aligned}
$$

respectively, have positive definite solutions $P_{1}>0$ and $P_{2}>0$, where the matrices $\hat{A}, \hat{C}, R$ are defined, respectively, in (3.17)-(3.19), then the filter (2.7) with parameters

$$
\begin{aligned}
K & =P_{2}^{-1}\left[\Theta^{T} R^{-1}+S U R^{-1 / 2}\right] \\
G & =\hat{A}-K \hat{C}
\end{aligned}
$$

where $\Theta$ is defined in (3.20), $U \in \mathbb{R}^{p \times p}$ is arbitrary orthogonal (i.e., $\left.U U^{T}=I\right), S \in \mathbb{R}^{n \times p}$ is an arbitrary matrix meeting $\Upsilon+$ $S S^{T}<0$, and $\Upsilon$ is defined in (3.22), will be such that the augmented system (2.15) is exponentially stable in the mean square for all admissible parameter uncertainties $\Delta A, \Delta A_{d}, \Delta C$ and the nonlinear disturbance input $f(x(t))$, independent of the unknown time-delay $h$.

Proof: First of all, it follows from Assumption 2.2 that $R^{-1}>0$ exists. Defining

$$
\begin{aligned}
\Sigma:= & A_{f}^{T} P+P A_{f} \\
& +P\left[\left(\varepsilon_{1}+\varepsilon_{2}\right) I+\varepsilon_{3} M_{f} M_{f}^{T}+\varepsilon_{4}^{-1} D_{f} D_{f}^{T}\right] P \\
& +\varepsilon_{1}^{-1} A_{d f}^{T} A_{d f}+\varepsilon_{3}^{-1} N_{f}^{T} N_{f}+\varepsilon_{4}\left(H F_{f}\right)^{T}\left(H F_{f}\right) \\
& +\lambda_{\max }\left(M_{d f}^{T} M_{d f}\right) \varepsilon_{2}^{-1} N_{d f}^{T} N_{d f} \\
:= & {\left[\begin{array}{cc}
\Sigma_{11} & \Sigma_{12} \\
\Sigma_{12}^{T} & \Sigma_{22}
\end{array}\right] }
\end{aligned}
$$

and setting

$$
P=\left[\begin{array}{cc}
P_{1} & 0 \\
0 & P_{2}
\end{array}\right]>0
$$

we have

$$
\begin{aligned}
\Sigma_{11}= & A^{T} P_{1}+P_{1} A \\
& +P_{1}\left[\left(\varepsilon_{1}+\varepsilon_{2}\right) I+\varepsilon_{3} M_{1} M_{1}^{T}+\varepsilon_{4}^{-1} D D^{T}\right] P_{1} \\
& +2 \varepsilon_{1}^{-1} A_{d}^{T} A_{d}+\varepsilon_{3}^{-1} N_{1}^{T} N_{1}+\varepsilon_{4} H^{T} H \\
& +\lambda_{\max }\left(M_{d f}^{T} M_{d f}\right) \varepsilon_{2}^{-1} N_{2}^{T} N_{2} \\
\Sigma_{12}= & (A-G-K C)^{T} P_{2} \\
& +P_{1}\left[\varepsilon_{3} M_{1}\left(M_{1}-K M_{2}\right)^{T}+\varepsilon_{4}^{-1} D D^{T}\right] P_{2} \\
\Sigma_{22}= & G^{T} P_{2}+P_{2} G \\
& +P_{2}\left[\left(\varepsilon_{1}+\varepsilon_{2}\right) I+\varepsilon_{3}\left(M_{1}-K M_{2}\right)\left(M_{1}-K M_{2}\right)^{T}\right. \\
& \left.\quad+\varepsilon_{4}^{-1} D D^{T}\right] P_{2} .
\end{aligned}
$$

It follows directly from (3.21) that $\Sigma_{11}<0$. By resorting to $G=\hat{A}-K \hat{C}$ and the definitions of $R$ and $\Theta$, we have

$$
\begin{aligned}
\Sigma_{22}= & (\hat{A}-K \hat{C})^{T} P_{2}+P_{2}(\hat{A}-K \hat{C}) \\
& +P_{2}\left[\left(\varepsilon_{1}+\varepsilon_{2}\right) I+\varepsilon_{3} M_{1} M_{1}^{T}\right. \\
& \quad-\varepsilon_{3} M_{1} M_{2}^{T} K^{T}-\varepsilon_{3} K M_{2} M_{1}^{T} \\
& \left.\quad+\varepsilon_{3} K M_{2} M_{2}^{T} K^{T}+\varepsilon_{4}^{-1} D D^{T}\right] P_{2} \\
= & \hat{A}^{T} P_{2}+P_{2} \hat{A} \\
& +P_{2}\left[\left(\varepsilon_{1}+\varepsilon_{2}\right) I+\varepsilon_{3} M_{1} M_{1}^{T}+\varepsilon_{4}^{-1} D D^{T}\right] P_{2} \\
& -\left(P_{2} K\right)\left(\hat{C}+\varepsilon_{3} M_{2} M_{1}^{T} P_{2}\right) \\
& -\left(\hat{C}+\varepsilon_{3} M_{2} M_{1}^{T} P_{2}\right)^{T}\left(P_{2} K\right)^{T} \\
& +\left(P_{2} K\right)\left(\varepsilon_{3} M_{1} M_{2}^{T}\right)\left(P_{2} K\right)^{T} \\
= & \hat{A}^{T} P_{2}+P_{2} \hat{A} \\
& +P_{2}\left[\left(\varepsilon_{1}+\varepsilon_{2}\right) I+\varepsilon_{3} M_{1} M_{1}^{T}+\varepsilon_{4}^{-1} D D^{T}\right] P_{2} \\
& -\left(P_{2} K\right) \Theta-\Theta^{T}\left(P_{2} K\right)^{T}+\left(P_{2} K\right) R\left(P_{2} K\right)^{T} \\
= & \hat{A}^{T} P_{2}+P_{2} \hat{A} \\
& +P_{2}\left[\left(\varepsilon_{1}+\varepsilon_{2}\right) I+\varepsilon_{3} M_{1} M_{1}^{T}+\varepsilon_{4}^{-1} D D^{T}\right] P_{2} \\
& -\Theta^{T} R^{-1} \Theta+\left[\left(P_{2} K\right) R^{1 / 2}-\Theta^{T} R^{-1 / 2}\right] \\
& \cdot\left[\left(P_{2} K\right) R^{1 / 2}-\Theta^{T} R^{-1 / 2}\right]^{T} . \\
&
\end{aligned}
$$

In the light of (3.23) and the orthogonality of $U$, it is easy to see that

$$
\begin{aligned}
& {\left[\left(P_{2} K\right) R^{1 / 2}-\Theta^{T} R^{-1 / 2}\right]\left[\left(P_{2} K\right) R^{1 / 2}-\Theta^{T} R^{-1 / 2}\right]^{T}} \\
& \quad=(S U)(S U)^{T}=S S^{T} .
\end{aligned}
$$


Considering the definition of $\Theta$ in (3.20), it follows from (3.30) and (3.31) that

$$
\begin{aligned}
\Sigma_{22}= & \left(\hat{A}-\varepsilon_{3} M_{1} M_{2}^{T} R^{-1} \hat{C}\right)^{T} P_{2} \\
& +P_{2}\left(\hat{A}-\varepsilon_{3} M_{1} M_{2}^{T} R^{-1} \hat{C}\right) \\
& +P_{2}\left[\left(\varepsilon_{1}+\varepsilon_{2}\right) I+\varepsilon_{3} M_{1} M_{1}^{T}+\varepsilon_{4}^{-1} D D^{T}\right. \\
& \left.\quad-\varepsilon_{3}^{2} M_{1} M_{2}^{T} R^{-1} M_{2} M_{1}^{T}\right] P_{2} \\
& -\hat{C}^{T} R^{-1} \hat{C}+S S^{T}=\Upsilon+S S^{T}
\end{aligned}
$$

where $\Upsilon$ is defined in (3.22). Recall that $S \in \mathbb{R}^{n \times p}$ is an arbitrary matrix meeting $\Upsilon+S S^{T}<0$. Then, (3.22) leads to $\Sigma_{22}<0$.

Moreover, substituting (3.24) into (3.28) immediately yields $\Sigma_{12}=0$, and therefore, $\Sigma<0$. Finally, it follows from Theorem 3.1 that the augmented system (2.15) is exponentially stable in the mean square for all admissible parameter uncertainties $\Delta A, \Delta A_{d}, \Delta C$ and nonlinear disturbance input $f(x(t))$, independent of the unknown time-delay $h$. This proves Theorem 3.2.

Remark 3.3: Theorem 3.2 provides a quadratic matrix inequality (QMI) approach to the design of robust filters for a class of nonlinear uncertain time-delay systems. When we cope with the QMIs (3.21) and (3.22), the local numerical searching algorithms suggested in [2] and [11] are effective for a relatively low-order model. With respect to the general existence conditions of the positive definite solutions to the QMIs and relevant algorithms, see [27]. It is seen that the existence of a positive definite solution to (3.21) means that the system matrix $A$ must be asymptotically stable, i.e., Assumption 2.1 holds. More specifically, since the QMIs (3.21) and (3.22) have the similar form, we now briefly discuss the conditions for the existence of the positive definite solutions to the QMI (3.21). It is easily accessible from [14] that there exists a positive definite solution to QMI (3.21) if and only if

$$
\left\|\Gamma^{1 / 2}(s I-A)^{-1} \Lambda^{1 / 2}\right\|_{\infty}<1
$$

where

$$
\begin{aligned}
\Gamma:= & 2 \varepsilon_{1}^{-1} A_{d}^{T} A_{d}+\varepsilon_{3}^{-1} N_{1}^{T} N_{1}+\varepsilon_{4} H^{T} H \\
& +\lambda_{\max }\left(M_{d f}^{T} M_{d f}\right) \varepsilon_{2}^{-1} N_{2}^{T} N_{2} \\
\Lambda:= & \left(\varepsilon_{1}+\varepsilon_{2}\right) I+\varepsilon_{3} M_{1} M_{1}^{T}+\varepsilon_{4}^{-1} D D^{T} .
\end{aligned}
$$

Remark 3.4: Note that there exist many free design parameters in the expression of expected filters. For example, we can choose free parameters $S$ meeting $\Upsilon+S S^{T}<0$ and orthogonal matrix $U$ in (3.23). Therefore, the set of the desired filter parameters, when it is not empty, must be very large, and much explicit freedom is subsequently offered. This gives the possibility for directly achieving further performance requirements on the filtering process such as the transient property, $\mathrm{H}_{2}$-norm constraint, and reliability behavior, which requires further investigations. It is remarkable that in [16], a similar freedom on an arbitrary orthogonal matrix in the parameterization of the set of filters was successfully employed to minimize the $\mathrm{H}_{2}$ norm of the filtering error transfer function by solving an unconstrained parametric optimization problem over the set of filters.

\section{NUMERICAL SIMULATION}

In this section, for the purpose of illustrating the usefulness and flexibility of the theory developed in this paper, we present a simulation example, focus on the steady-state exponentially filtering and proceed to determine the filter parameters.

Consider the nonlinear uncertain stochastic state-delayed system (2.1) and (2.2) with parameters as follows:

$$
\begin{aligned}
& A=\left[\begin{array}{ccc}
-2.5 & 0.2 & -0.2 \\
-0.3 & -3 & -0.4 \\
1.5 & -0.4 & -5
\end{array}\right] \\
& A_{d}=\left[\begin{array}{rrc}
0.03 & 0.01 & 0.01 \\
0.01 & -0.04 & 0 \\
-0.01 & 0.01 & -0.02
\end{array}\right] \\
& D=\left[\begin{array}{cc}
0 & 0.1 \\
0.1 & 0 \\
0.2 & 0.2
\end{array}\right], \quad E_{1}=\left[\begin{array}{l}
0.1 \\
0.1 \\
0.1
\end{array}\right] \\
& E_{2}=\left[\begin{array}{l}
0.01 \\
0.01 \\
0.01
\end{array}\right], \quad C=\left[\begin{array}{lll}
1 & 0 & 0 \\
0 & 1 & 0 \\
0 & 0 & 1
\end{array}\right] \\
& f(x)=\left[\begin{array}{l}
0.1 \sin x_{1} \\
0.1 \sin x_{2}
\end{array}\right], \quad M_{1}=\left[\begin{array}{ccc}
0.45 & 0 & 0.05 \\
0 & 0.45 & 0 \\
0.15 & 0 & 0.15
\end{array}\right] \\
& M_{2}=\left[\begin{array}{ccc}
0 & 0.65 & 0.05 \\
0.05 & 0 & 0.35 \\
0.28 & 0.18 & 0
\end{array}\right] \\
& N_{1}=\left[\begin{array}{ccc}
0.02 & 0.02 & 0 \\
0 & 0 & 0.02 \\
0 & 0.02 & 0
\end{array}\right] \\
& N_{2}=\left[\begin{array}{ccc}
0 & 0.06 & 0 \\
0 & 0 & 0.06 \\
0.02 & 0 & 0
\end{array}\right] \\
& H=\left[\begin{array}{ccc}
0.3 & 0 & 0.01 \\
0 & 0.2 & 0 \\
0.01 & 0 & 0.4
\end{array}\right] \\
& F(t)=\sin t I_{3}, \quad h=0.1, \quad \varphi(t)=0.1 .
\end{aligned}
$$

In this example, we are interested in designing a linear, delayless, uncertainty-independent state estimator (2.7) such that for all admissible uncertainties as well as the nonlinear disturbance input, the dynamics of the estimation error is stochastically exponentially stable in the mean square, independent of the time delay.

To show the flexibility of the proposed design method, we will discuss two cases by using the design freedom in choosing parameters $\varepsilon_{i}(i=1,2,3,4), S$ and $U$, as discussed in Remark 3.4 .

Case 1: We set $\varepsilon_{1}=0.1, \varepsilon_{2}=0.2, \varepsilon_{3}=0.6$, and $\varepsilon_{4}=$ 2.5. Then, we can obtain a positive definite solution $P_{1}$ to the 
quadratic matrix inequality (3.21) and, subsequently, the matrices $\hat{A}, \hat{C}$, and $R$, as follows:

$$
\begin{aligned}
P_{1} & =\left[\begin{array}{ccc}
13.9428 & 0.0181 & -7.3328 \\
0.0181 & 14.0227 & 2.0288 \\
-7.3328 & 2.0288 & 27.2244
\end{array}\right] \\
\hat{A} & =\left[\begin{array}{rrr}
-1.1179 & 0.3098 & 0.3116 \\
-0.3564 & -1.2239 & 0.0724 \\
1.8065 & -0.1672 & -3.7662
\end{array}\right] \\
\hat{C} & =\left[\begin{array}{lll}
0.9911 & 2.4701 & 0.4676 \\
0.0706 & 1.0735 & 0.8041 \\
0.8702 & 0.7340 & 1.2303
\end{array}\right] \\
R & =\left[\begin{array}{lll}
0.2550 & 0.0105 & 0.0702 \\
0.0105 & 0.0750 & 0.0084 \\
0.0702 & 0.0084 & 0.0665
\end{array}\right] .
\end{aligned}
$$

Furthermore, a positive definite solution to the quadratic matrix inequality (3.22) and the matrix $\Upsilon$ are calculated as

$$
\begin{gathered}
P_{2}=\left[\begin{array}{ccc}
16.9752 & 3.8009 & -0.9295 \\
3.8009 & 22.8890 & 4.1436 \\
-0.9295 & 4.1436 & 30.2289
\end{array}\right] \\
\Upsilon=\left[\begin{array}{ccc}
-0.4196 & -0.1362 & -0.2034 \\
-0.1362 & -1.3213 & -1.3975 \\
-0.2034 & -1.3975 & -2.6507
\end{array}\right] .
\end{gathered}
$$

Next, we choose the matrix $S$ meeting $\Upsilon+S S^{T}<0$ and an orthogonal matrix $U$ as

$$
S=0.6 I_{3}, \quad U=I_{3}
$$

and therefore, we obtain the expected filter parameters from (3.23) and (3.24) as the following:

$$
\begin{aligned}
& K=\left[\begin{array}{rlr}
-0.4468 & 0.0342 & 2.4116 \\
1.1347 & 0.5619 & -0.3067 \\
-0.3599 & 0.6406 & 1.3549
\end{array}\right], \\
& G=\left[\begin{array}{rrr}
-2.7760 & -0.3933 & -2.4740 \\
-1.2538 & -4.4048 & -0.5326 \\
0.9390 & -0.9603 & -5.7798
\end{array}\right]
\end{aligned}
$$

Denote the error states $e_{i}=x_{i}-\hat{x}_{i}(i=1,2,3)$. The responses of error dynamics to initial conditions are shown in Fig. 1, and the real state $x_{1}$ (respectively, $x_{2}, x_{3}$ ) and its estimate $\hat{x}_{1}$ (respectively, $\hat{x}_{2}, \hat{x}_{3}$ ) are displayed in Fig. 2 (respectively, Figs. 3 and 4). The simulation results imply that the desired goal is well achieved.

Case 2: In this case, we select $\varepsilon_{1}=0.3, \varepsilon_{2}=0.1, \varepsilon_{3}=0.8$, and $\varepsilon_{4}=4.5$, and then get

$$
\begin{aligned}
P_{1} & =\left[\begin{array}{ccc}
10.4585 & -0.0382 & -5.6468 \\
-0.0382 & 10.5764 & 1.7530 \\
-5.6468 & 1.7530 & 21.4049
\end{array}\right] \\
\hat{A} & =\left[\begin{array}{rrr}
-1.1255 & 0.3066 & 0.2408 \\
-0.3314 & -1.2553 & -0.0170 \\
1.8702 & -0.2612 & -4.2050
\end{array}\right]
\end{aligned}
$$

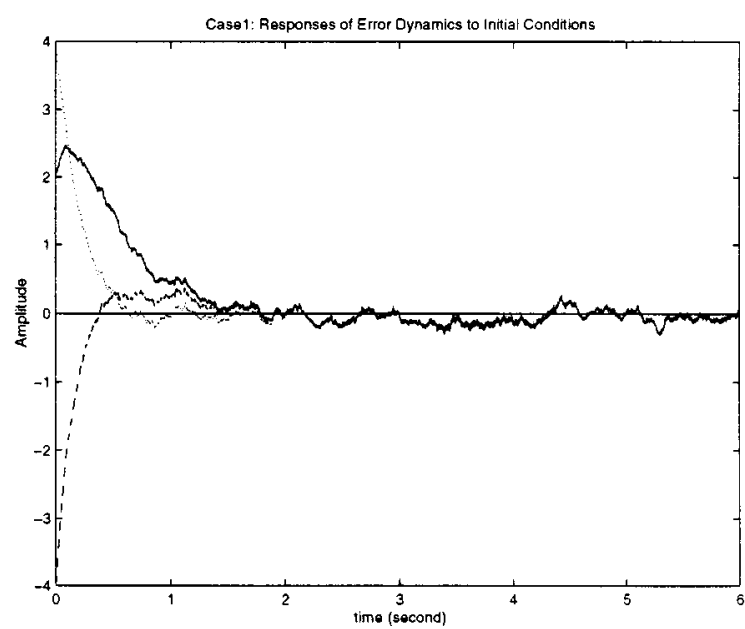

Fig. 1. $e_{1}$ (solid), $e_{2}$ (point), $e_{3}$ (dashed).

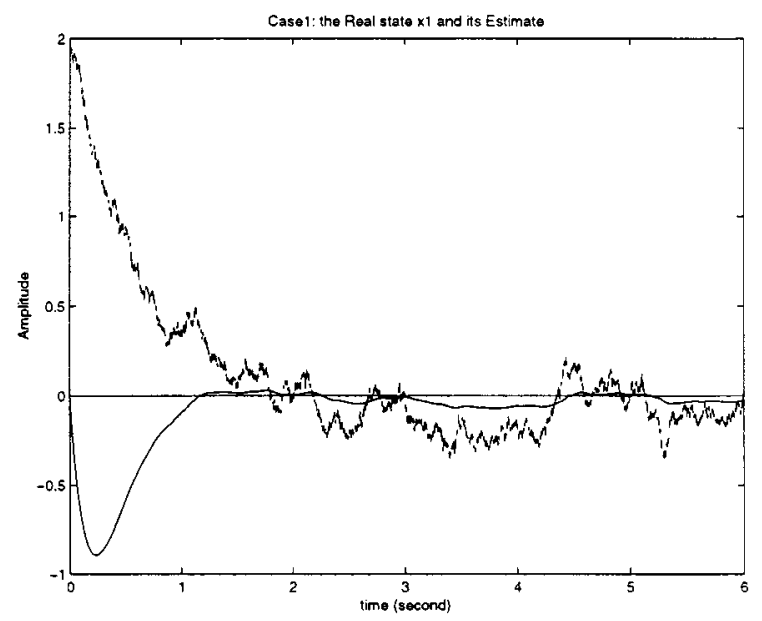

Fig. 2. $x_{1}$ (dashed), $\hat{x}_{1}$ (solid).

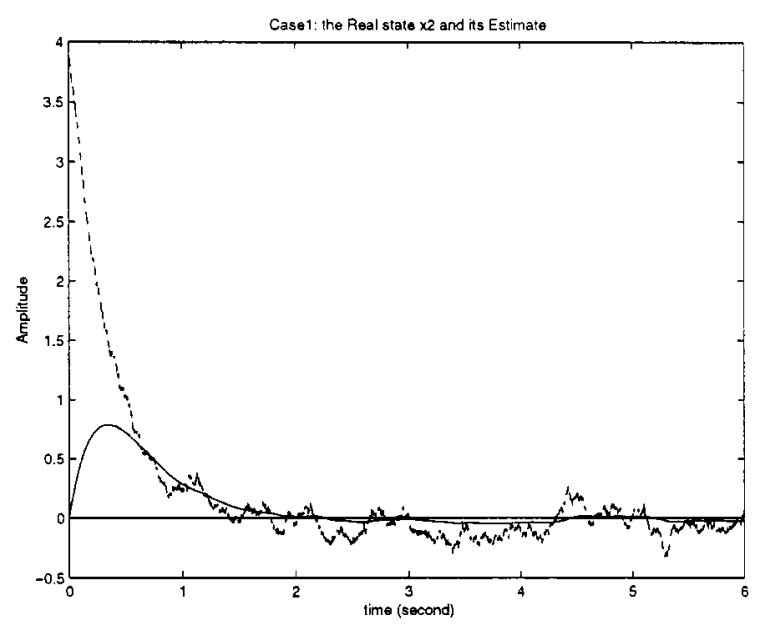

Fig. 3. $x_{2}$ (dashed), $\hat{x}_{2}$ (solid).

$$
\begin{aligned}
\hat{C} & =\left[\begin{array}{ccc}
0.9781 & 2.4853 & 0.5273 \\
0.0636 & 1.0829 & 0.8467 \\
0.8620 & 0.7404 & 1.2636
\end{array}\right] \\
P_{2} & =\left[\begin{array}{ccc}
12.8515 & 2.6431 & -0.9593 \\
2.6431 & 17.5561 & 4.0617 \\
-0.9593 & 4.0617 & 25.9338
\end{array}\right] .
\end{aligned}
$$




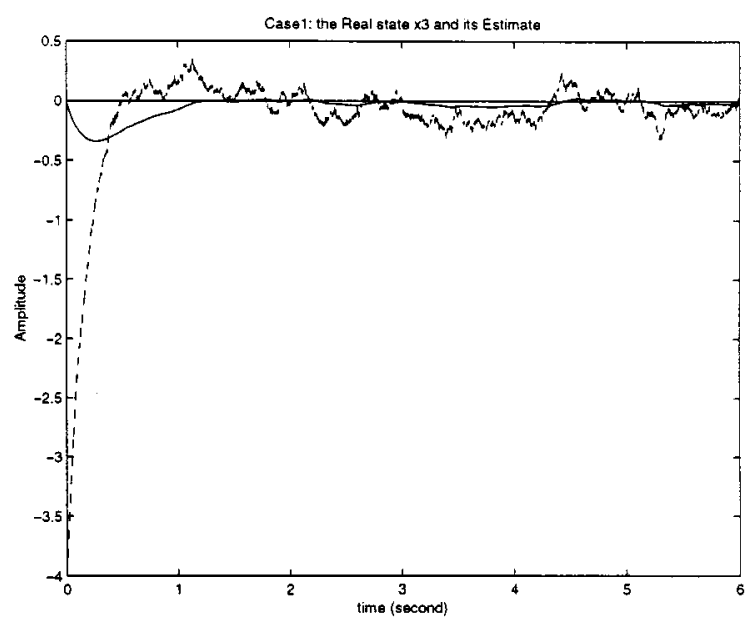

Fig. 4. $x_{3}$ (dashed), $\hat{x}_{3}$ (solid).

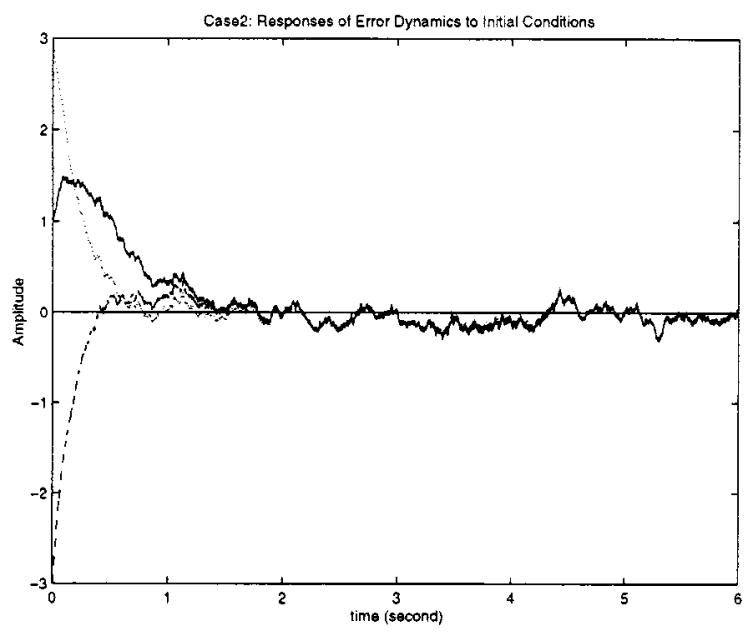

Fig. 5. $e_{1}$ (solid), $e_{2}$ (point), $e_{3}$ (dashed).

For this case, the matrix $S$ meeting $\Upsilon+S S^{T}<0$ and an orthogonal matrix $U$ are chosen as

$$
S=0.5 I_{3}, \quad U=-I_{3}
$$

and it follows from (3.23) and (3.24) that

$$
\begin{aligned}
& K=\left[\begin{array}{rlr}
-0.5973 & 0.0945 & 2.4471 \\
1.1558 & 0.3441 & -0.2764 \\
-0.3226 & 0.6482 & 1.1128
\end{array}\right] \\
& G=\left[\begin{array}{rlr}
-2.6567 & -0.1230 & -2.6164 \\
-1.2456 & -4.2959 & -0.5686 \\
1.1852 & -0.9852 & -5.9899
\end{array}\right] .
\end{aligned}
$$

For the error states $e_{i}=x_{i}-\hat{x}_{i}(i=1,2,3)$, the responses of error dynamics to initial conditions are shown in Fig. 5, and the real state $x_{1}$ (respectively, $x_{2}, x_{3}$ ) and its estimate $\hat{x}_{1}$ (respectively, $\hat{x}_{2}, \hat{x}_{3}$ ) are displayed in Fig. 6 (respectively, Figs. 7 and 8). The simulation results demonstrate that the estimation error is exponentially stable in the mean square, and thus, the prescribed performance requirements on the filtering process are guaranteed by the developed theory.

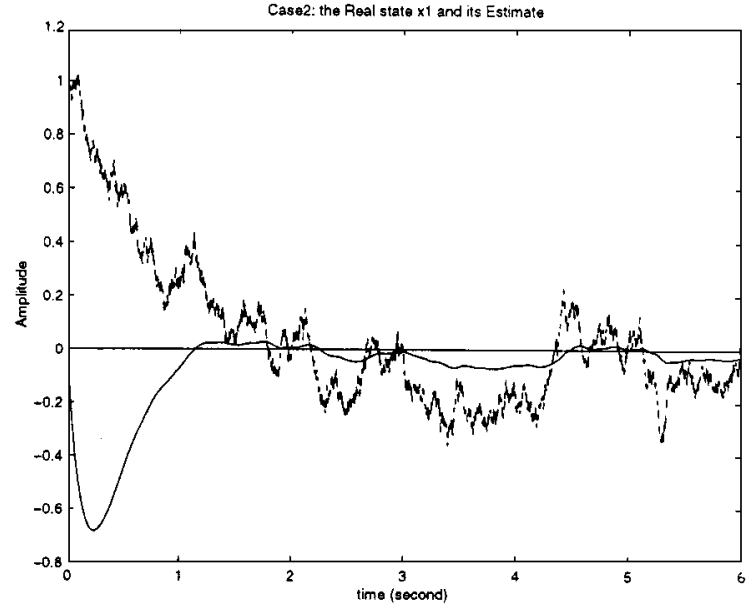

Fig. 6. $x_{1}$ (dashed), $\hat{x}_{1}$ (solid).

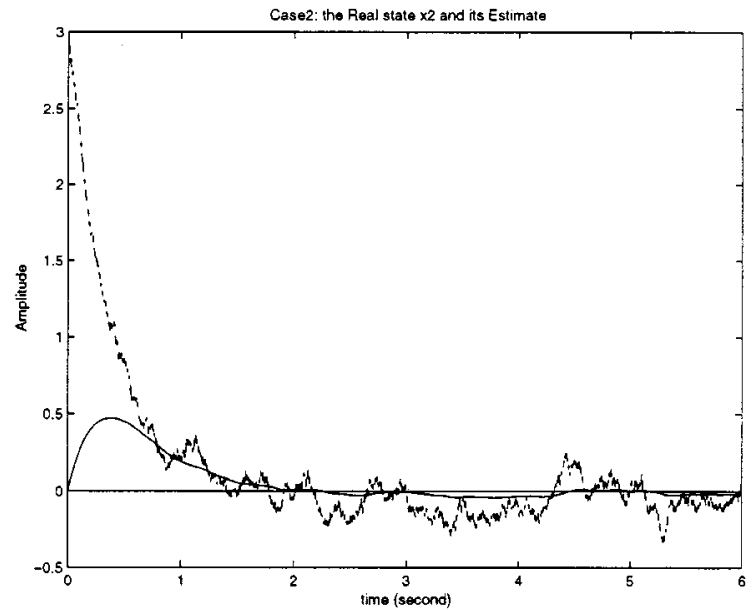

Fig. 7. $x_{2}$ (dashed), $\hat{x}_{2}$ (solid).

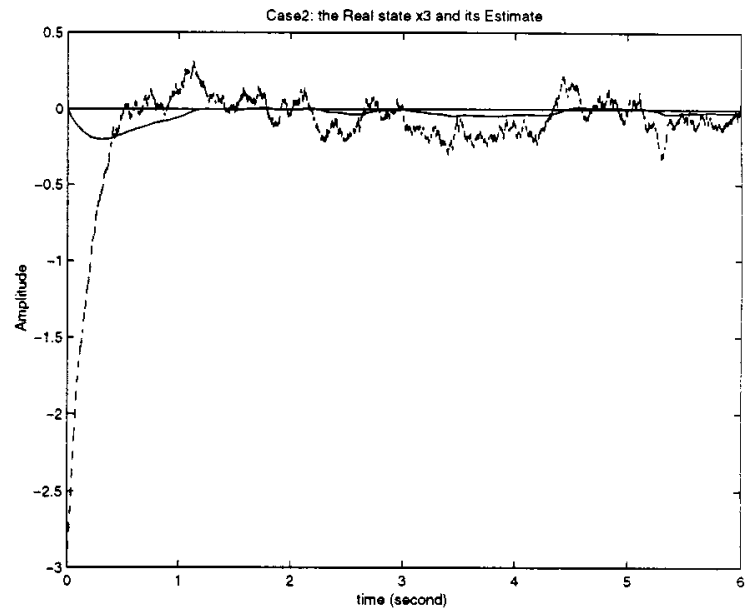

Fig. 8. $x_{3}$ (dashed), $\hat{x}_{3}$ (solid)

\section{CONCLUSIONS}

The problem of robust filtering for a class of nonlinear uncertain stochastic time-delay systems has been addressed in this paper. A linear filter has been designed to achieve the prescribed robust exponential stability constraints (in the mean square), regardless of the admissible parameter uncertainties, the bounded 
nonlinear disturbance input, and the unknown state delay. Both the filter analysis and design issues have been discussed in detail by means of quadratic matrix inequalities. The existence conditions as well as the analytical expression of desired filters have been parameterized. We have demonstrated that the desired robust exponential filters for this class of nonlinear time-delay systems, when they exist, are usually a large set, and the remaining freedom can be used to meet other expected performance requirements.

One of the future research topics is the development of efficient algorithms with guaranteed convergence. Finally, in our opinion, the idea introduced in this paper can also be applied to design robust filters for more complex systems such as sampled-data systems and stochastic parameter systems.

\section{ACKNOWLEDGMENT}

The first author is grateful to Prof. H. Unbehauen of Ruhr University Bochum, Germany, for detailed comments and to Prof. D. Prätzel-Wolters of the University of Kaiserslautern, Germany, for helpful suggestions. The authors would like to thank the four anonymous referees for their valuable suggestions and constructive comments that have improved the presentation.

\section{REFERENCES}

[1] B. D. O. Anderson and J. B. Moore, Optimal Filtering. Englewood Cliffs, NJ: Prentice-Hall, 1979.

[2] E. Beran and K. Grigoriadis, "A combined alternating projections and semidefinite programming algorithm for low-order control design," in Proc. 13th IFAC World Congr., vol. C, San Francisco, CA, 1996, pp. $85-90$.

[3] D. S. Bernstein and W. M. Haddad, "Steady-state Kalman filtering with an $H_{\infty}$ error bound," Syst. Contr. Lett., vol. 12, pp. 9-16, 1989.

[4] P. Bolzern, P. Colaneri, and G. De Nicolao, "Optimal robust filtering with time-varying parameter uncertainty," Int. J. Contr., vol. 63, pp. $557-576,1996$.

[5] _ - "Finite escapes and convergence properties of guaranteed-cost robust filters," Automatica, vol. 33, pp. 31-47, 1997.

[6] R. C. Chung and P. R. Belanger, "Minimum-sensitivity filter for linear time-invariant stochastic systems with uncertain parameter," IEEE Trans. Automat. Contr., vol. AC-21, pp. 98-100, 1976.

[7] C. E. de Souza, U. Shaked, and M. Fu, "Robust $H_{\infty}$ filtering for continuous time varying uncertain systems with deterministic input signal," IEEE Trans. Signal Processing, vol. 43, pp. 709-719, Mar. 1995.

[8] C. E. de Souza and U. Shaked, "Robust $H_{2}$ filtering for uncertain systems with measurable inputs," IEEE Trans. Signal Processing, vol. 47 , pp. 2286-2292, Aug. 1999.

[9] M. Fu, C. E. de Souza, and L. Xie, " $H_{\infty}$ estimation for uncertain systems," Int. J. Robust Nonlinear Contr., vol. 2, pp. 87-105, 1992.

[10] J. C. Geromel, "Optimal linear filtering under parameter uncertainty," IEEE Trans. Signal Processing, vol. 47, pp. 168-175, Jan. 1999.

[11] J. C. Geromel, P. L. D. Peres, and S. R. Souza, "Output feedback stabilization of uncertain systems through a min/max problem," in Proc. 12th IFAC World Congr., vol. 6, Sydney, Australia, 1993, pp. 35-38.

[12] M. J. Grimble, " $H_{\infty}$ design of optimal linear filters," in Linear Circuits, Systems and Signal Processing: Theory and Applications, C. I. Byrnes, C. F. Martin, and R. E. Saeks, Eds. Amsterdam, The Netherlands: North-Holland, 1988, pp. 533-540.

[13] F. Hsiao and S. Pan, "Robust Kalman filter synthesis for uncertain multiple time-delay stochastic systems," J. Dyn. Syst. Meas. Contr., vol. 118, pp. 803-808, 1996.

[14] P. P. Khargonekar, I. R. Petersen, and K. Zhou, "Robust stabilization of uncertain linear system: Quadratic stabilizability and $H_{\infty}$ control theory," IEEE Trans. Automat. Contr., vol. 35, pp. 356-361, 1990.

[15] $\mathrm{H}$. Li and M. Fu, "Linear matrix inequality approach to robust $H_{\infty}$ filtering," IEEE Trans. Signal Processing, vol. 45, pp. 2338-2350, Sept. 1997.
[16] L. Li, L. Xie, W.-Y. Yan, and Y. C. Soh, "Design of low-order linearphase IIR filters via orthogonal projection," IEEE Trans. Signal Processing, vol. 47, pp. 434-448, Feb. 1999.

[17] M. S. Mahmoud, N. F. Al-Muthairi, and S. Bingulac, "Robust Kalman filtering for continuous time-lag systems," Syst. Contr. Lett., vol. 38, pp. 309-319, 1999.

[18] M. Malek-Zavarei and M. Jamshidi, Time-Delay Systems: Analysis, Optimization and Application. Amsterdam, The Netherlands, 1987.

[19] X. Mao, "Robustness of exponential stability of stochastic differential delay equations," IEEE Trans. Automat. Contr., vol. 41, pp. 442-447, 1996.

[20] - Stochastic Differential Equations and Applications. London, U.K.: Ellis Horwood, 1997.

[21] K. M. Nagpal and P. P. Khargonekar, "Filtering and smoothing in an $H_{\infty}$ setting," IEEE Trans. Automat. Contr., vol. 36, pp. 152-166, 1991.

[22] S.-I. Niculescu, E. I. Verriest, L. Dugard, and J. M. Dion, "Stability and robust stability of time-delay systems: A guided tour," in Stability and Control of Time-Delay Systems, L. Dugard et al., Eds. Berlin, Germany: Springer, 1998, vol. 228, pp. 1-71.

[23] J. O'Reilly, Observers for Linear Systems. New York: Academic, 1983.

[24] I. R. Petersen and D. C. McFarlane, "Robust state estimation for uncertain systems," in Proc. 30th Conf. Decision Contr., Brighton, U.K., 1991, pp. 2630-2631.

[25] — "Optimal guaranteed cost control and filtering for uncertain linear systems," IEEE Trans. Automat. Contr., vol. 39, pp. 1971-1977, 1994.

[26] K. Reif and R. Unbehauen, "The extended Kalman filter as an exponential observer for nonlinear systems," IEEE Trans. Signal Processing, vol. 47, pp. 2324-2328, Aug. 1999.

[27] A. Saberi, P. Sannuti, and B. M. Chen, " $H_{2}$ optimal control," in Series in Systems and Control Engineering. London, U.K.: Prentice-Hall Int., 1995.

[28] U. Shaked, " $H_{\infty}$ minimum error state estimation of linear stationary processes," IEEE Trans. Automat. Contr., vol. 35, pp. 554-558, 1990.

[29] U. Shaked and C. E. de Souza, "Robust minimum variance filtering," IEEE Trans. Signal Processing, vol. 43, pp. 2474-2483, Sept. 1995.

[30] U. Shaked and Y. Theodor, " $H_{\infty}$ optimal estimation: A tutorial," in Proc. IEEE Conf. Decision Contr., Tucson, AZ, 1992, pp. 2278-2286.

[31] Z. Wang and H. Unbehauen, "Robust state estimation for perturbed continuous systems with circular pole and error variance constraints," Int. J. Contr., vol. 69, pp. 383-392, 1998.

[32] — - "Robust $\mathrm{H}_{2} / \mathrm{H}_{\infty}$-state estimation for systems with error variance constraints: The continuous-time case," IEEE Trans. Automat. Contr., vol. 44, pp. 1061-1065, 1999.

[33] Z. Wang, J. Zhu, and H. Unbehauen, "Robust filter design with timevarying parameter uncertainty and error variance constraints," Int. J. Contr., vol. 72, pp. 30-38, 1999.

[34] Z. Wang, B. Huang, and H. Unbehauen, "Robust $H_{\infty}$ observer design of linear state delayed systems with parametric uncertainty: The discrete-time case," Automatica, vol. 35, pp. 1161-1167, 1999.

[35] Z. Wang and B. Huang, "Robust $H_{2} / H_{\infty}$ filtering for linear systems with error variance constraints," IEEE Trans. Signal Processing, vol. 48, pp. 2463-2467, Aug. 2000.

[36] L. Xie and Y. C. Soh, "Robust Kalman filtering for uncertain systems," Syst. Contr. Lett., vol. 22, pp. 123-129, 1994.

[37] E. Yaz, "On the almost sure and mean-square exponential convergence of some stochastic observers," IEEE Trans. Automat. Contr., vol. 35, pp. 935-936, 1990.

[38] E. Yaz, "Robust, exponentially fast state estimator for some nonlinear stochastic systems," Int. J. Syst. Sci., vol. 23, pp. 557-567, 1992.

[39] E. Yaz and A. Azemi, "Observer design for discrete and continuous nonlinear stochastic systems," Int. J. Syst. Sci., vol. 24, pp. 2289-2302, 1993.

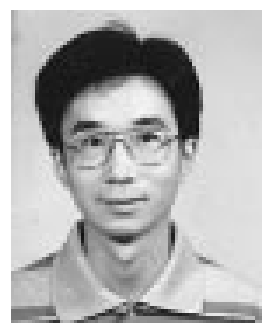

Zidong Wang (M'00) was born in Gaoyou, China, on February 21, 1966. He received the B.Sci. degree in mathematics in 1986 from Suzhou University, Suzhou, China, the M.Sci. degree in applied mathematics in 1988 from Donghua University (formerly China Textile University), Shanghai, and the Ph.D. degree in electrical engineering in 1994 from Nanjing University of Science and Technology, Nanjing, China.

He was appointed as Lecturer at East China Institute of Technology in 1990 and was promoted to 
Associate Professor at Nanjing University of Science and Technology in 1994. From January 1997 to December 1998, he was an Alexander von Humboldt Research Fellow at the Automatic Control Laboratory, Ruhr University Bochum, Bochum, Germany. In January 1999, he joined the Department of Mathematics, University of Kaiserslautern, Kaiserslautern, Germany, as a Faculty Staff Member (Lecturer). His research interests include filtering and control for uncertain systems, stochastic systems, nonlinear systems, and sampled-data systems and their applications. He has published more than 60 papers in refereed international journals.

Dr. Wang was awarded the JSPS Research Fellowship in August 1998 from Japan Society for the Promotion of Science. He was a recipient of the Outstanding Science and Technology Development Award from National Education Committee of China (twice in 1996 and once in 1998) and the National Science Investigator Award from the National Natural Science Foundation of China in 1995. He was nominated the outstanding reviewer for the journal Automatica for 2000 and received Standing Membership of the Technical Committee on Control of the International Association of Science and Technology for Development, also in 2000.

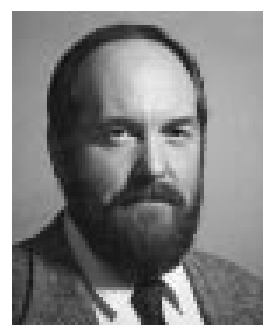

Keith J. Burnham received the B.Sci. degree in mathematics, the M.Sci. degree in control engineering, and the $\mathrm{Ph} . \mathrm{D}$. degree in adaptive control, all from Coventry University, Coventry, U.K., in 1981, 1984 , and 1991, respectively.

$\mathrm{He}$ has been a Professor of Industrial Control Systems with the School of Mathematical and Information Sciences, Coventry University, and Director of the University's Control Theory and Applications Centre, since 1999. This is a multidisciplinary research center in which effective collaboration takes place amongst staff from across the University. There are currently a number of research programs with U.K.-based industrial organizations, many of which are involved with the design and implementation of adaptive control systems.

Dr. Burnham is an Associate Member of the Institution of Electrical Engineers, a Member of the Institute of Mathematics and its Applications, and a Member of the Institute of Measurement and Control. 